\title{
Combination of a pulmonary recruitment maneuver and intraperitoneal bupivacaine for the reduction of postoperative shoulder pain in gynecologic laparoscopy: a randomized, controlled trial
}

\author{
Minae Cho, MD ${ }^{1 *}$, Chul Jung Kim, MD ${ }^{2 *}$,Tae Soo Hahm, MD, PhD ${ }^{3}$, Yoo-Young Lee, MD ${ }^{1}$, Tae-Joong Kim, MD ${ }^{1}$ \\ Jeong-Won Lee, MD¹, Byoung-Gie Kim, MD, PhD, Duk-Soo Bae, MD, PhD¹, Chel Hun Choi, MD, PhD ${ }^{1}$

\begin{abstract}
Department of Obstetrics and Gynecology, ${ }^{1}$ Samsung Medical Center, Sungkyunkwan University School of Medicine, Seoul, ${ }^{2}$ College of Medicine, Konyang University, Daejeon; ${ }^{3}$ Department of Anesthesiology and Pain Medicine, Samsung Medical Center, Sungkyunkwan University School of Medicine, Seoul, Korea
\end{abstract}

\section{Objective}

To compare the efficacy of a pulmonary recruitment maneuver using lower airway pressure $\left(30 \mathrm{~cm}_{2} \mathrm{O}\right)$ and intraperitoneal bupivacaine, alone or in combination, for reducing shoulder pain after gynecologic laparoscopy.

Methods

A prospective controlled study was performed in a teaching hospital with patients who underwent elective gynecologic laparoscopic surgery. Two hundred eighty-seven patients were randomized into 1 of 4 groups: group $A$, placebo; group $B$, intraperitoneal instillation of bupivacaine; group $C_{1} \mathrm{CO}_{2}$ removal by a pulmonary recruitment maneuver; group D, combination of intraperitoneal bupivacaine and pulmonary recruitment maneuver. The interventions were performed at the end of surgery. Shoulder pain was recorded on a visual analog scale (VAS) at 1, 6, 12 , and 24 hours postoperatively.

Results

The overall incidence of shoulder pain was $49.8 \%$ and the incidence tended to gradually decrease from group $A$ to group $D(59.0 \%$ in group $A, 54.8 \%$ in group $B, 44.4 \%$ in group $C$, and $41.5 \%$ in group $D ; P=0.026)$. In addition, the VAS scores gradually decreased from group $A$ to $D$, although a statistically significant difference was only found at 6 hours postoperatively $(P=0.03)$. There were no complications related to the interventions.

\section{Conclusion}

The combination of a pulmonary recruitment maneuver with intraperitoneal bupivacaine significantly reduced shoulder pain after gynecologic laparoscopy.

\section{Trial Registration}

ClinicalTrials.gov Identifier: NCT01039441.

Keywords: Gynecologic laparoscopy; Intraperitoneal bupivacaine; Pulmonary recruitment maneuver; Shoulder pain; VAS score

\section{Introduction}

Although the pain following laparoscopic surgery is usually milder and easier to resolve than that after a laparotomy $[1,2]$, shoulder pain is a very common presentation that may cause even more discomfort than the pain at the incision site. The
Received: 2019.08.30. Revised: 2019.10.31. Accepted: 2019.11.03. Corresponding author: Chel Hun Choi, MD, PhD

Department of Obstetrics and Gynecology, Samsung Medical Center, Sungkyunkwan University School of Medicine, 81 Irwonro, Gangnam-gu, Seoul 06351, Korea

E-mail: chelhun.choi@samsung.com https://orcid.org/0000-0002-0199-6669

*These two authors contributed equally to this paper.

Articles published in Obstet Gynecol Sci are open-access, distributed under the terms of the Creative Commons Attribution Non-Commercial License (http://creativecommons org/licenses/by-nc/3.0/) which permits unrestricted non-commercial use, distribution, and reproduction in any medium, provided the original work is properly cited.

Copyright $\odot 2020$ Korean Society of Obstetrics and Gynecology 


\title{
Obstetrics \& Gynecology Science
}

\author{
Vol. 63, No. 2, 2020
}

incidence of shoulder pain following laparoscopy ranges from $35 \%$ to $65 \%$ and the severity of pain varies [3-6]. The pain intensity peaks during the first few hours after surgery and usually declines over the ensuing $2-3$ days $[7,8]$.

Even though it remains unclear why a considerable number of patients experience shoulder pain after laparoscopy, peritoneal stretching and diaphragmatic irritation caused by $\mathrm{CO}_{2}$ gas seem to play a significant role in its mechanism [9-12]. Thus, several trials have assessed the use of intraperitoneal local anesthetics with controversial results [13-20].

In 2008, Phelps et al. [21] reported that a pulmonary recruitment maneuver that removed residual abdominal $\mathrm{CO}_{2}$ after laparoscopy reduced shoulder pain by more than half. The maneuver they used consisted of 5 manual pulmonary inflations with a peak pressure of $60 \mathrm{~cm} \mathrm{H}_{2} \mathrm{O}$. Although there were no adverse pulmonary effects, the risk of pneumothorax associated with high airway pressure remains questionable.

The purpose of this randomized controlled trial was to compare the effectiveness of pulmonary recruitment maneuver with relatively lower airway pressure $\left(30 \mathrm{~cm} \mathrm{H}_{2} \mathrm{O}\right)$ and subdiaphragmatic instillation of bupivacaine, either alone or in combination, for minimizing shoulder pain after gynecologic laparoscopy.

\section{Materials and methods}

The information regarding clinical trial registration was posted on ClinicalTrials.gov (url: http://www.clinicaltrials.gov, registration identifier: NCT01039441). Patients presenting to our institution for elective gynecologic laparoscopic surgery were asked to participate and informed consent was obtained. Patients with scheduled laparoscopic surgery for benign adnexal disease were eligible for the study. The age of enrolled patients was between 15 and 65 years. The exclusion criteria were as follows: 1) conversion to a laparotomy, 2) an operative time of more than 3 hours, and 3) serious adverse effects which made it impossible to score pain.

Patients were randomly assigned at a 1:1:1:1 ratio to the $A, B, C$, or D groups, according to a computer-generated random number-selected list that had been set prior to the initiation of the trial. The surgeons and patients were blinded to the group allocation. The group allocation was known only to the anesthesiologist and scrub nurse for the specific case until the end of the entire surgical procedure.

A standardized, general anesthetic regimen was applied to every patient. The induction of anesthesia was performed with Fresofol $(2 \mathrm{mg} / \mathrm{kg})$ and fentanyl $(1.5 \mu \mathrm{g} / \mathrm{kg})$. Rocuronium was used as a muscle relaxant to facilitate endotracheal intubation. Along with mechanical ventilation, the anesthesia was sustained with a continuous infusion of Fresofol and fentanyl.

With an infraumbilical Veress needle, the distension medium $\left(\mathrm{CO}_{2}\right)$ was introduced into the abdominal cavity. A 5-mm trocar was inserted at the infraumbilical area when abdominal pressure reached $>10 \mathrm{mmHg}$. A 0-degree laparoscope was introduced through the trocar. Two additional 5-mm and one 10-mm trocars were inserted under direct visualization in the left/right iliac fossa and suprasymphyseal midline, respectively. The intra-abdominal pressure was monitored throughout the procedure and was maintained at a maximum pressure of $15 \mathrm{mmHg}$.

The patient was placed in the Trendelenburg position (30 degrees) after completion of the surgery. According to the group allocation, one of four procedures was performed. $50 \mathrm{~mL}$ of normal saline was instilled under the diaphragm for the patients in group A (control). The patients in group B had a mixed solution ( $0.5 \%$ bupivacaine $20 \mathrm{~mL}+$ normal saline $30 \mathrm{~mL}$ ) instilled under the diaphragm. In group C, after applying $50 \mathrm{~mL}$ of normal saline using the same method, $\mathrm{CO}_{2}$ was removed by performing a pulmonary recruitment maneuver. The procedure consists of 5 manual lung inflations in which the peak pressure was less than $30 \mathrm{~cm} \mathrm{H}_{2} \mathrm{O}$. The fifth positive pressure inflation lasted for approximately 5 seconds. While the anesthesiologist was performing the pulmonary recruitment maneuver, the surgeon was instructed to open the trocar valve fully to remove intraperitoneal $\mathrm{CO}_{2}$ gas. Subdiaphragmatic instillation of the mixed solution ( $0.5 \%$ bupivacaine $20 \mathrm{~mL}+$ normal saline $30 \mathrm{~mL}$ ) in combination with the pulmonary recruitment maneuver was applied to patients in group D. During the procedure, saline or bupivacaine was sprayed subdiaphragmatically via a laparoscopic approach.

Postoperative analgesia was provided with intravenous ketolac/demerol when a patient complained of postoperative pain greater than 4 on a numeric pain rating scale. Postoperative patient-controlled analgesia (PCA) was not applied and routine non-steroidal anti-inflammatory drugs (NSAIDs) were administered when the patient restarted oral feeding.

Questionnaires asking about shoulder pain were distrib- 


\title{
Obstetrics \& Gynecology Science
}

\author{
Minae Cho, et al. Shoulder pain after laparoscopy
}

uted to patients after each surgery. Patients completed the questionnaires by describing the frequency and severity of pain during the first 24 hours after the surgery using a visual analog scale (VAS). The patients themselves marked a point along the visual scale ranging from 0 (no pain) to 10 (extreme pain) that best described their pain at 1, 6, 12, and 24 hours postoperatively. In addition, the investigators recorded 5 parameters on the case report forms: operation time, blood loss, duration of hospitalization, use of analgesics, and incidence of postoperative events.

With the pain score as the primary endpoint, the sample size calculation was based on the assumption that the pain score (VAS) would be different between groups at any postoperative timepoint $(1,6,12$, and 24 hour $)$ and the difference in pain score would be lower by 0.5 in groups $B$ and $C$ and lower by 1.0 in group $D$ than in group $A$ (control) with SDs $(1.30,0.75,0.74$, and 1.20 , respectively, in a previous study). Therefore, 59 patients per group were allocated to the 4 groups at a significance level of 0.05 and $90 \%$ power to detect any difference.

In this study, a per-protocol analysis was adopted, because dropouts were expected due to the possibility of conversion to laparotomy for various surgical reasons. The KruskalWallis test for continuous variables and $\chi^{2}$ test for categorical variables were used to analyze differences between patient groups. A P-value of less than 0.05 was regarded as statistically significant. The analysis in this trial was performed using SPSS for Windows, version 15.0 (SPSS Inc., Chicago, IL, USA).

\section{Results}

Between December 2008 and July 2009, 291 patients agreed to participate in the clinical trial. Of those who were excluded from the final analysis, 4 patients were not randomized due to lost randomization identification, 10 patients were converted to laparotomy, 7 patients had severe endometriosis, 4 patients had an operative time of more than 3 hours, and 15 patients did not submit responses to the questionnaire (Fig. 1). Therefore, 251 randomly assigned patients with analyzable data (61 in group A, 62 in group B, 63 in group $C$, and 65 in group $D$ ) were obtained. The patients' characteristics were similar with respect to age, body mass index (BMI), type of surgery, operative time, blood loss, and length of hospital stay (Table 1).

Overall, 49.8\% (125 of 251) of patients reported postoperative shoulder pain during the first 24 hours. The incidence of shoulder pain tended to gradually decrease from group $A$

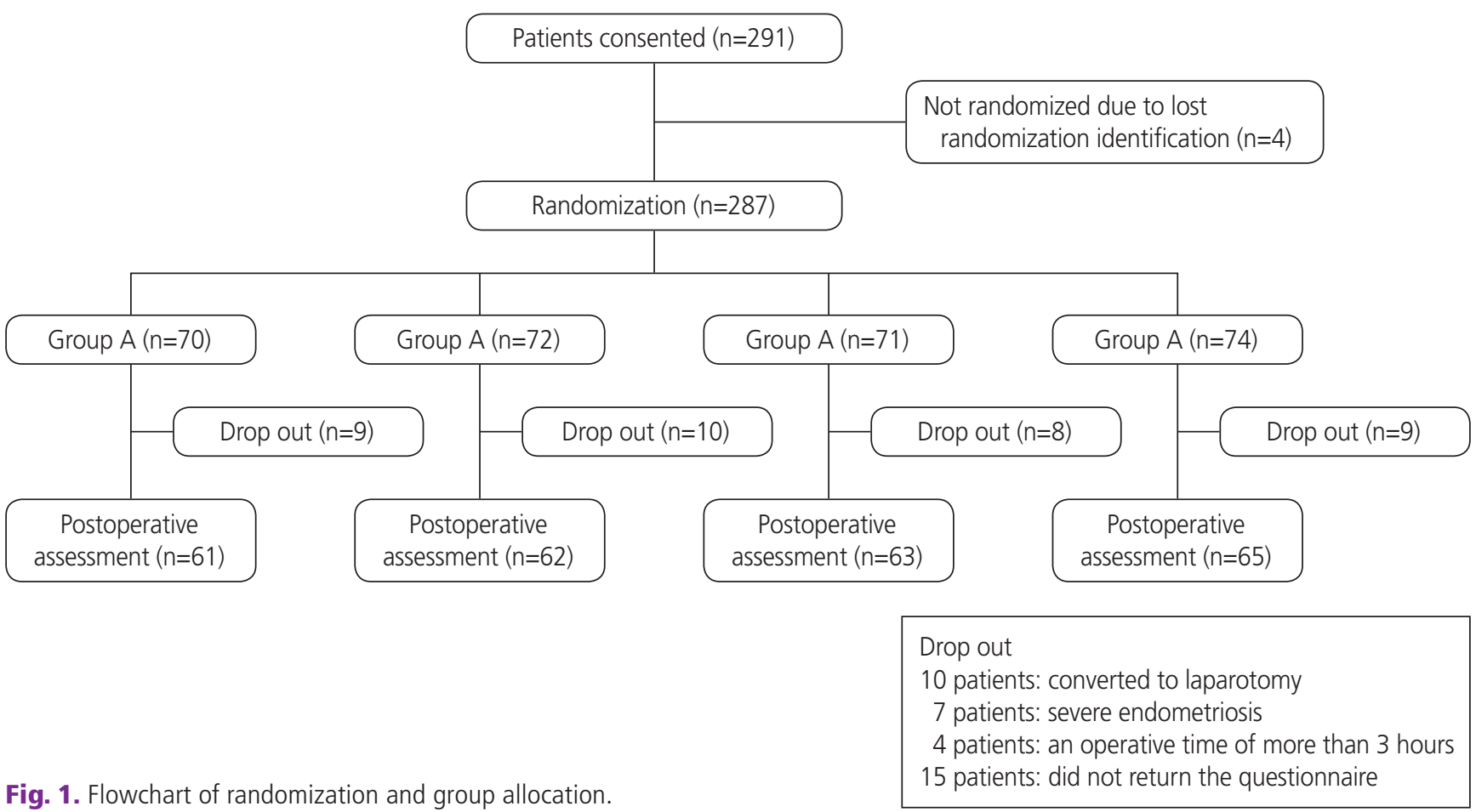




\section{Obstetrics \& Gynecology Science}

Vol. 63, No. 2, 2020

to group $D(59.0 \%$ in group $A, 54.8 \%$ in group $B, 44.4 \%$ in group $C$, and $41.5 \%$ in group $D ; P=0.026$; Table 1 ).

The postoperative pain scores are shown in Table 2 and Fig. 2. The highest VAS score was 6 hours after surgery and the scores tended to decrease in the following hours among all groups. The VAS scores tended to be gradually decrease from group $A$ to $D$ at any postoperative time interval. However, only at 6 hours postoperatively was there a statistically significant difference $(P=0.03)$. Patients in group $D$ reported significantly less shoulder pain than group $A$ at 1 hour (1.02 \pm 1.59 vs. $1.74 \pm 1.97, P=0.021), 6$ hours $(1.18 \pm 1.74$ vs. $2.23 \pm 2.30, P=0.005)$, and 12 hours $(0.88 \pm 1.45$ vs. $1.61 \pm 1.99, P=0.032$ ) postoperatively.

The 4 groups were similar in terms of the incidence of nausea and vomiting after surgery. No complications related to the interventions were noted. Cardiovascular or pulmonary complications were not found as a result of the maneuver.

\section{Discussion}

This study was performed to assess the efficacy of a pulmonary recruitment maneuver and intraperitoneal instillation of bupivacaine to reduce shoulder pain after gynecologic laparoscopy. Compared with the control group, the VAS scores for shoulder pain tended to gradually decrease from groups $B$ to $D$ at any of the postoperative time intervals. Of interest, the combination therapy group (group D) showed the most significant decrease in shoulder pain at 1, 6, and 12 hours postoperatively.

For the binomial analysis, we combined the groups according to the intervention; pulmonary recruitment maneuver

Table 1. Patient characteristics $(n=251)$

\begin{tabular}{|c|c|c|c|c|c|}
\hline Characteristic & $\begin{array}{c}\text { Group A } \\
(n=61)\end{array}$ & $\begin{array}{c}\text { Group B } \\
(n=62)\end{array}$ & $\begin{array}{c}\text { Group C } \\
(n=63)\end{array}$ & $\begin{array}{c}\text { Group D } \\
(n=65)\end{array}$ & $P$-value ${ }^{\text {a) }}$ \\
\hline Age (yr) & $41.2 \pm 12.4$ & $37.8 \pm 12.0$ & $38.5 \pm 12.4$ & $38.7 \pm 10.6$ & 0.42 \\
\hline Body mass index $\left(\mathrm{kg} / \mathrm{m}^{2}\right)$ & $22.4 \pm 2.7$ & $23.1 \pm 3.4$ & $22.9 \pm 2.8$ & $23.4 \pm 3.6$ & 0.69 \\
\hline Type of surgery & & & & & 0.96 \\
\hline Ovarian cystectomy & 30 & 35 & 31 & 32 & \\
\hline Salpingo-oophorectomy & 26 & 23 & 26 & 29 & \\
\hline Others & 5 & 4 & 6 & 4 & \\
\hline Diagnosis & & & & & 0.99 \\
\hline Mature cystic teratoma & 18 & 20 & 20 & 17 & \\
\hline Endometriosis & 20 & 18 & 23 & 23 & \\
\hline Benign cystadenoma & 15 & 16 & 15 & 17 & \\
\hline Others & 8 & 8 & 5 & 8 & \\
\hline Previous abdominal surgery & $23(37.7 \%)$ & $20(32.3 \%)$ & $22(34.9 \%)$ & $24(36.9 \%)$ & 0.92 \\
\hline Adhesion & & & & & 0.79 \\
\hline Present & 22 & 23 & 20 & 19 & \\
\hline Absent & 39 & 39 & 43 & 45 & \\
\hline Operative time (min) & $68.6 \pm 28.8$ & $68.9 \pm 29.7$ & $66.8 \pm 25.5$ & $61.5 \pm 23.6$ & 0.37 \\
\hline Mean blood loss (mL) & $69.3 \pm 54.1$ & $68.9 \pm 44.8$ & $62.9 \pm 42.9$ & $68.6 \pm 41.6$ & 0.84 \\
\hline Hospital stay (hr) & $49.3 \pm 12.2$ & $47.6 \pm 13.5$ & $46.3 \pm 13.2$ & $50.3 \pm 14.2$ & 0.31 \\
\hline Shoulder pain & & & & & $0.026^{b)}$ \\
\hline Present & 36 & 34 & 28 & 27 & \\
\hline Absent & 25 & 28 & 35 & 38 & \\
\hline
\end{tabular}

Group A, placebo; group B, intraoperative intraperitoneal bupivacaine administration alone; group C, pulmonary recruitment maneuver; and group $D$, intraoperative intraperitoneal bupivacaine administration combined with pulmonary recruitment maneuver.

${ }^{\text {a) }}$ Kruskall-Wallis test or $\chi^{2}$ test; ${ }^{b)}$ Linear-by-Linear Association. 


\section{Obstetrics \& Gynecology Science}

Minae Cho, et al. Shoulder pain after laparoscopy

(groups A and B vs. groups $C$ and D), and intraperitoneal bupivacaine (groups $A$ and $C$ vs. groups $B$ and $D$ ). The postoperative pain scores were significantly lower in the pulmonary recruitment maneuver group (groups $C$ and D) compared with those in the control group (groups $A$ and $B$ ) at 1 hours (1.04 \pm 1.62 vs. $1.50 \pm 1.72, P=0.03), 6$ hours $(1.34 \pm 1.89$ vs. $2.03 \pm 2.22, P=0.007)$, and 12 hours $(1.01 \pm 1.58$ vs. $1.52 \pm 1.95, P=0.03)$ after surgery. However, there was no statistically significant difference between the intraperitoneal bupivacaine group (groups $A$ and $C$ ) and control group (groups B and D) (data not shown).

One of the major limitations of the study is that we cannot explain the precise pathophysiology of postoperative shoulder pain. It is widely accepted that phrenic nerve stimulation causes referred pain of $\mathrm{C} 4$, which projects to the shoulder $[9,11,22]$. The irritation of the phrenic nerve might be caused by two main etiologies consisting of distension of the diaphragm $[10,11]$ and residual intra-abdominal $\mathrm{CO}_{2}$ gas that could induce intra-abdominal acidosis $[9,23,24]$. Therefore, diverse maneuvers have been suggested to minimize the shoulder pain caused by $\mathrm{CO}_{2}$-related irritation of the phrenic nerve [25-30]. For example, for the relief of phrenic nerve damage, the use of sub-diaphragmatically administered local anesthetics have been studied, providing conflicting results [13-19]. Furthermore, several studies have suggested that evacuation of intraperitoneal $\mathrm{CO}_{2}$ gas at the end of surgery $[22,31]$, either by draining or aspirating remaining gas, significantly reduced the severity of shoulder pain and use of postoperative analgesics $[3,23,25]$.

The suboptimal effect of bupivacaine in our study might be due to the timing of the administration of the local anesthetic. One study claimed that the timing of the administration of local anesthetic agent is crucial to reducing postoperative pain [32]. In their study, those who received intraperitoneal bupivacaine immediately after establishment of pneumoperitoneum reported significantly lower pain scores than those who received it at the end of surgery. Another explanation

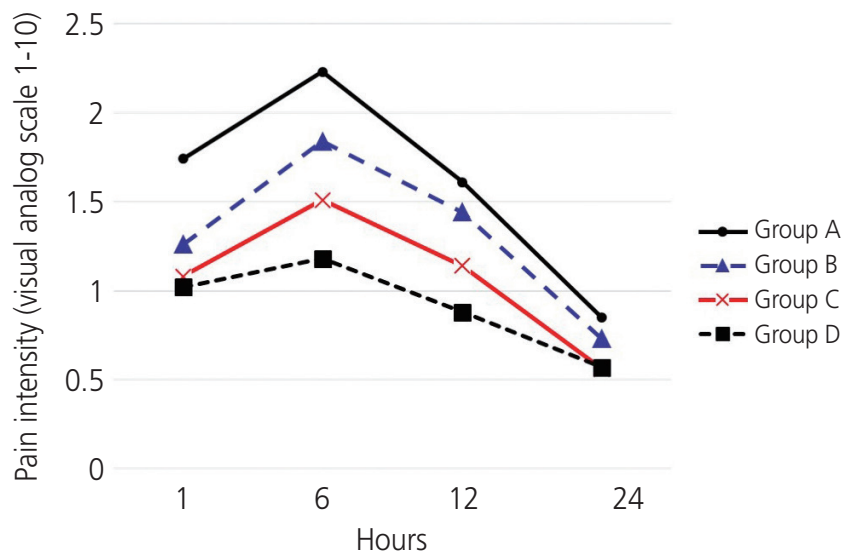

Fig. 2. Intensity of shoulder pain recorded using a visual analogue scale (VAS) at 1, 6, 12, and 24 hours after surgery in group A (control); group B (intraperitoneal instillation of bupivacaine alone); group C (pulmonary recruitment maneuver alone); and group D (intraperitoneal instillation of bupivacaine combined with pulmonary recruitment maneuver).

Table 2. Intensity of shoulder pain recorded on a visual analog scale (VAS) at each postoperative time interval $(n=251)$

\begin{tabular}{|c|c|c|c|c|c|c|}
\hline \multirow{2}{*}{ Time interval } & & \multicolumn{4}{|c|}{ VAS score $(95 \% \mathrm{CI})$} & \multirow{2}{*}{$P$-value ${ }^{\text {a) }}$} \\
\hline & & Group A $(n=61)$ & Group B $(n=62)$ & Group C (n=63) & Group D $(n=65)$ & \\
\hline \multirow[t]{2}{*}{1 hour } & Mean \pm SD & $1.74 \pm 1.97$ & $1.26 \pm 1.79$ & $1.08 \pm 1.67$ & $1.02 \pm 1.59$ & 0.08 \\
\hline & Median (IQR) & $1(0-3)$ & $0(0-2.75)$ & $0(0-2)$ & $0(0-2)$ & \\
\hline \multirow[t]{2}{*}{6 hours } & Mean $\pm S D$ & $2.23 \pm 2.30$ & $1.84 \pm 2.14$ & $1.51 \pm 2.03$ & $1.18 \pm 1.74$ & 0.03 \\
\hline & Median (IQR) & $2(0-4)$ & $1(0-3.75)$ & $0(0-3)$ & $0(0-3)$ & \\
\hline \multirow[t]{2}{*}{12 hours } & Mean $\pm S D$ & $1.61 \pm 1.99$ & $1.44 \pm 1.92$ & $1.14 \pm 1.70$ & $0.88 \pm 1.45$ & 0.14 \\
\hline & Median (IQR) & $0(0-3)$ & $0(0-2.75)$ & $0(0-2)$ & $0(0-2)$ & \\
\hline \multirow[t]{2}{*}{24 hours } & Mean $\pm S D$ & $0.85 \pm 1.46$ & $0.73 \pm 1.33$ & $0.56 \pm 1.12$ & $0.57 \pm 1.03$ & 0.76 \\
\hline & Median (IQR) & $0(0-1)$ & $0(0-1)$ & $0(0-0.5)$ & $0(0-1)$ & \\
\hline
\end{tabular}

Group A, placebo; group B, intraperitoneal instillation of bupivacaine alone; group C, pulmonary recruitment maneuver; and group D, intraperitoneal instillation of bupivacaine combined with pulmonary recruitment maneuver.

$\mathrm{Cl}$, confidence interval; SD, standard deviation; IQR, interquartile range.

${ }^{\text {a) }}$ Kruskall-Wallis test 


\section{Obstetrics \& Gynecology Science}

Vol. 63, No. 2, 2020

for our findings may be that the duration of pain suppression was limited; the half-life of bupivacaine is 3.5 hours with 3- to 10-hours duration of action, such that no benefit would be expected with respect to shoulder pain up to 24 hours after surgery. In this study, $50 \mathrm{~mL}$ of saline was instilled under the diaphragm as a control for the bupivacaine group. Tsimoyiannis et al. [33] suggested that instillation of normal saline under the diaphragm induced significantly less shoulder pain after laparoscopic cholecystectomy. Presumably, the decrease in $\mathrm{CO}_{2}$ between the liver and diaphragm resulted in limited irritation [33]. Thus, the reduced efficacy of bupivacaine herein may have been due to the intervention in control group (A and C), which also had similar or some efficacy in reducing shoulder pain. Therefore, there was no statistically significant difference between the bupivacaine group ( $B$ and $D$ ) and control group ( $A$ and $C$ ) in the binomial analysis in this study.

The pulmonary recruitment maneuver in the current study, as proposed by Phelps et al. [21], has some definite advantages; the maneuver is readily available without extra devices or resources and is time-saving compared to previous techniques. When positive pressure ventilation is applied, it inflates the lungs, which in turn, lowers the diaphragm. Therefore, increased intraperitoneal pressure forces the $\mathrm{CO}_{2}$ gas to escape the peritoneal cavity. The decrease in intra-abdominal acidosis results in diminished phrenic nerve or peritoneal irritation [21]. In this study, the maximum pressure of the pulmonary recruitment maneuver was $30 \mathrm{~cm} \mathrm{H}_{2} \mathrm{O}$, which was lower than that reported by Phelps et al. $\left(60 \mathrm{~cm} \mathrm{H}_{2} \mathrm{O}\right)$ [21]. In Phelps's study, cardiovascular or pulmonary complications related to the pulmonary recruitment maneuver were not reported. However, inflation with a maximum pressure of $60 \mathrm{~cm} \mathrm{H}_{2} \mathrm{O}$ may carry a risk of pneumothorax. In fact, the literature suggests that a pulmonary recruitment maneuver with a peak pressure of $40 \mathrm{~cm} \mathrm{H}_{2} \mathrm{O}$ is a safe and effective way of improving arterial oxygenation during anesthesia [34-38]. It is meaningful that our study showed that the pulmonary recruitment maneuver significantly reduces shoulder pain in more than triple the number of patients and using a lower pressure than that in the study of Phelps et al. [21].

One of major limitations of our study is its relatively high dropout rate. 40 out of 291 patients were excluded due to a longer operation time, conversion, severe endometriosis, loss of identification, or for not responding to the questionnaire. Even though we anticipated dropouts due to surgical con- version, the high dropout rate resulted in a minor disparity between the number of patients in each group.

There were no statistically significant differences in postoperative pain, analgesic use, nausea, or vomiting in this study. This may be because of the routine use of oral NSAIDs and an oral prokinetic agent (mosapride). Previous studies have shown that the intervention group, when compared to the control group, showed a significantly lower incidence of postoperative nausea and vomiting. Moreover, the frequency of analgesic use was significantly lower in patients receiving intraperitoneal bupivacaine $[21,32]$. Routine medication might mask the effect of the intervention on those abovementioned measures. Since patients were freely given extra painkillers on demand, the inability to control postoperative analgesic use was one of our study limitations. However, since no significant difference in postoperative analgesic use between study groups was found, we can assume that the result of the investigation is still reliable.

In conclusion, our study demonstrates that performing a pulmonary recruitment maneuver at the completion of laparoscopic surgery significantly reduces shoulder pain after gynecologic laparoscopy. Its use in combination with the intraperitoneal instillation of bupivacaine further decreased shoulder pain.

\section{Acknowledgements}

This clinical trial was supported by a grant of the Korea Health 21 R\&D Project, Ministry of Health \& Welfare, Republic of Korea (0412-CR01-0704-0001).

\section{Conflict of interest}

No potential conflict of interest relevant to this article was reported.

\section{Ethical approval}

The protocol of the prospective clinical trial was approved by the Institutional Review Board (IRB) of Samsung Medical Center (IRB file No. 2008-07-071) and performed in accordance with the principles of the Declaration of Helsinki. 


\section{Obstetrics \& Gynecology Science}

Minae Cho, et al. Shoulder pain after laparoscopy

\section{Patient consent}

Written informed consents were obtained.

\section{References}

1. Grace PA, Quereshi A, Coleman J, Keane R, McEntee G, Broe $\mathrm{P}$, et al. Reduced postoperative hospitalization after laparoscopic cholecystectomy. Br J Surg 1991;78:160-2.

2. Ortega $A E$, Hunter JG, Peters JH, Swanstrom LL, Schirmer B; Laparoscopic Appendectomy Study Group. A prospective, randomized comparison of laparoscopic appendectomy with open appendectomy. Am J Surg 1995;169:208-12.

3. Alexander JI. Pain after laparoscopy. Br J Anaesth 1997;79:369-78

4. Cason CL, Seidel SL, Bushmiaer M. Recovery from laparoscopic cholecystectomy procedures. AORN J 1996;63:1099-103.

5. Madsen MR, Jensen KE. Postoperative pain and nausea after laparoscopic cholecystectomy. Surg Laparosc Endosc 1992;2:303-5.

6. Tsai HW, Wang PH, Yen MS, Chao KC, Hsu TF, Chen YJ. Prevention of postlaparoscopic shoulder and upper abdominal pain: a randomized controlled trial. Obstet Gynecol 2013;121:526-31.

7. Ure BM, Troidl H, Spangenberger W, Dietrich A, Lefering R, Neugebauer E. Pain after laparoscopic cholecystectomy. Intensity and localization of pain and analysis of predictors in preoperative symptoms and intraoperative events. Surg Endosc 1994;8:90-6.

8. Lee $\mathrm{DH}$, Song $\mathrm{T}$, Kim KH, Lee KW. Incidence, natural course, and characteristics of postlaparoscopic shoulder pain. Surg Endosc 2018;32:160-5.

9. Jackson SA, Laurence AS, Hill JC. Does post-laparoscopy pain relate to residual carbon dioxide? Anaesthesia 1996;51:485-7.

10. Coventry DM. Anaesthesia for laparoscopic surgery. J R Coll Surg Edinb 1995;40:151-60.

11. Korell M, Schmaus F, Strowitzki T, Schneeweiss SG, Hepp H. Pain intensity following laparoscopy. Surg Laparosc Endosc 1996;6:375-9.

12. Song T, Kim KH, Lee KW. The intensity of postlaparoscopic shoulder pain is positively correlated with the amount of residual pneumoperitoneum. J Minim Invasive Gynecol 2017;24:984-989.e1.

13. Labaille T, Mazoit JX, Paqueron X, Franco D, Benhamou $D$. The clinical efficacy and pharmacokinetics of intraperitoneal ropivacaine for laparoscopic cholecystectomy. Anesth Analg 2002;94:100-5.

14. Lee IO, Kim SH, Kong MH, Lee MK, Kim NS, Choi YS, et al. Pain after laparoscopic cholecystectomy: the effect and timing of incisional and intraperitoneal bupivacaine. Can J Anaesth 2001;48:545-50.

15. Narchi P, Benhamou D, Fernandez H. Intraperitoneal local anaesthetic for shoulder pain after day-case laparoscopy. Lancet 1991;338:1569-70.

16. Kelly MC. An assessment of the value of intraperitoneal bupivacaine for analgesia after laparoscopic sterilisation. Br J Obstet Gynaecol 1996;103:837-9.

17. Benhamou D, Narchi P, Mazoit JX, Fernandez H. Postoperative pain after local anesthetics for laparoscopic sterilization. Obstet Gynecol 1994;84:877-80.

18. Loughney AD, Sarma V, Ryall EA. Intraperitoneal bupivacaine for the relief of pain following day case laparoscopy. Br J Obstet Gynaecol 1994;101:449-51.

19. Shaw IC, Stevens J, Krishnamurthy $S$. The influence of intraperitoneal bupivacaine on pain following major laparoscopic gynaecological procedures. Anaesthesia 2001;56:1041-4.

20. Kaloo P, Armstrong S, Kaloo C, Jordan V. Interventions to reduce shoulder pain following gynaecological laparoscopic procedures. Cochrane Database Syst Rev 2019:CD011101.

21. Phelps P, Cakmakkaya OS, Apfel CC, Radke OC. A simple clinical maneuver to reduce laparoscopy-induced shoulder pain: a randomized controlled trial. Obstet Gynecol 2008;111:1155-60.

22. Mouton WG, Bessell JR, Otten KT, Maddern GJ. Pain after laparoscopy. Surg Endosc 1999;13:445-8.

23. Fredman B, Jedeikin R, Olsfanger D, Flor P, Gruzman A. Residual pneumoperitoneum: a cause of postoperative pain after laparoscopic cholecystectomy. Anesth Analg 1994;79:152-4.

24. Alexander JI, Hull MG. Abdominal pain after laparoscopy: the value of a gas drain. Br J Obstet Gynaecol 1987;94:267-9.

25. Abbott J, Hawe J, Srivastava P, Hunter D, Garry R. Intraperitoneal gas drain to reduce pain after laparoscopy: 


\section{Obstetrics \& Gynecology Science}

Vol. 63, No. 2, 2020

randomized masked trial. Obstet Gynecol 2001;98:97-100.

26. Esmat ME, Elsebae MM, Nasr MM, Elsebaie SB. Combined low pressure pneumoperitoneum and intraperitoneal infusion of normal saline for reducing shoulder tip pain following laparoscopic cholecystectomy. World J Surg 2006;30:1969-73.

27. Nursal TZ, Yildirim S, Tarim A, Noyan T, Poyraz P, Tuna $N$, et al. Effect of drainage on postoperative nausea, vomiting, and pain after laparoscopic cholecystectomy. Langenbecks Arch Surg 2003;388:95-100.

28. Palmes D, Röttgermann S, Classen C, Haier J, Horstmann R. Randomized clinical trial of the influence of intraperitoneal local anaesthesia on pain after laparoscopic surgery. Br J Surg 2007;94:824-32.

29. Swift G, Healey M, Varol N, Maher P, Hill D. A prospective randomised double-blind placebo controlled trial to assess whether gas drains reduce shoulder pain following gynaecological laparoscopy. Aust N Z J Obstet Gynaecol 2002:42:267-70.

30. Tsai HW, Chen YJ, Ho CM, Hseu SS, Chao KC, Tsai SK, et al. Maneuvers to decrease laparoscopy-induced shoulder and upper abdominal pain: a randomized controlled study. Arch Surg 2011;146:1360-6.

31. Rettenmaier MA, Micha JP, Lopez KL, Wilcox AM, Goldstein $\mathrm{BH}$. A prospective, observational trial assessing the efficacy of abdominal compression in reducing laparoscopic-induced shoulder pain. Surg Innov 2017;24:552-6.

32. Pasqualucci $A$, de Angelis V, Contardo R, Colò F, Terrosu
G, Donini A, et al. Preemptive analgesia: intraperitoneal local anesthetic in laparoscopic cholecystectomy. A randomized, double-blind, placebo-controlled study. Anesthesiology 1996;85:11-20.

33. Tsimoyiannis EC, Siakas P, Tassis A, Lekkas ET, Tzourou $H$, Kambili M. Intraperitoneal normal saline infusion for postoperative pain after laparoscopic cholecystectomy. World J Surg 1998;22:824-8.

34. Tusman G, Böhm SH, Vazquez de Anda GF, do Campo $J \mathrm{~L}$, Lachmann B. 'Alveolar recruitment strategy' improves arterial oxygenation during general anaesthesia. $\mathrm{Br} J \mathrm{An}$ aesth 1999;82:8-13.

35. Magnusson L, Tenling A, Lemoine R, Högman $M$, Tydén $H$, Hedenstierna $G$. The safety of one, or repeated, vital capacity maneuvers during general anesthesia. Anesth Analg 2000;91:702-7.

36. Ricard JD. Barotrauma during mechanical ventilation: why aren't we seeing any more? Intensive Care Med 2004;30:533-5.

37. Ryu K, Choi W, Shim J, Song T. The impact of a pulmonary recruitment maneuver to reduce post-laparoscopic shoulder pain: a randomized controlled trial. Eur J Obstet Gynecol Reprod Biol 2017;208:55-60.

38. Sharami SH, Sharami MB, Abdollahzadeh M, Keyvan A. Randomised clinical trial of the influence of pulmonary recruitment manoeuvre on reducing shoulder pain after laparoscopy. J Obstet Gynaecol 2010;30:505-10. 